\title{
Reliability of testicular stiffness quantification using shear wave elastography in predicting male fertility: a preliminary prospective study
}

\author{
Alpaslan Yavuz ${ }^{1}$, Adem Yokus$^{1}, K_{\text {Kerem Taken}}^{2}$, Abdussamet Batur ${ }^{1}$, Mesut Ozgokce1, \\ Harun Arslan ${ }^{1}$
}

${ }^{1}$ Department of Radiology, ${ }^{2}$ Department of Urology, Van Yuzuncu Yil University, Medical Faculty, Van, Turkey

\begin{abstract}
:
Aims: To evaluate the reliability of testicular stiffness quantification using shear wave elastography in predicting the fertility potential of males and for the pre-diagnosis of disorders based upon sperm quantification. Material and methods: One hundred males between the ages of 19-49 years (mean age of 28.77 \pm 6.11 ), ninety of whom with complaints of infertility, were enrolled in this prospective study. Scrotal grey-scale, Doppler ultrasound (US), and mean testicular shear wave velocity quantifications (SWVQs) were performed. The volumes of testes, as well as the grade of varicocele if present, were recorded. The mean shear wave velocity values (SWVVs) of each testis and a mean testicular SWVV for each patient were calculated. The semen-analyses of patients were consecutively performed. Results: There were significant negative correlations between the mean testicular SWVVs of patients and their sperm counts or the testis volumes $(r=-0.399, r=-0.565 ; p<0.01$, respectively). A positive correlation was found between testicular volumes and sperm counts $(r=0.491, p<0.01)$. The cut-off values regarding mean testicular SWVV to distinguish normal sperm count from azoospermia and oligozoospermia were $1.465 \mathrm{~m} / \mathrm{s}(75.0 \%$ sensitivity and $75.0 \%$ specificity) and $1.328 \mathrm{~m} / \mathrm{s}(64.3 \%$ sensitivity and $68.2 \%$ specificity), respectively, and the value to distinguish oligozoospermia from azoospermia was $1.528 \mathrm{~m} / \mathrm{s}$ (66.7\% sensitivity, $60.7 \%$ specificity). Conclusion: The mean testicular SWVQ using the ARFI shear wave technique was a reliable, non-invasive and acceptably stable method for predicting male infertility, especially related to sperm count issues.
\end{abstract}

Keywords: shear wave elastography; ultrasound; testis; infertility; semen analysis

\section{Introduction}

Ultrasound (US) elastography is a cutting-edge imaging modality that facilitates the evaluation of parenchymal viscoelasticity by quantifying the stiffness of soft tissues. This technique is an easily accessible, non-invasive ultrasound technique with real-time results that can be divided into two subgroups: one in which the force is ap-

Received 10.09.2017 Accepted 20.12.2017

Med Ultrason

2018, Vol. 20, No 2, 141-147

Corresponding author: Dr. Alpaslan Yavuz, MD, Assoc. Prof. Chief of Radiology Department, Yuzuncu Yil University, Medical Faculty 65100, Van, Turkey

Phone: +90 5323424959 (mobile)

Fax: +90 4322168352

E-mail: dralpyavuz@hotmail.com plied to the tissue within the region of interest (ROI) by manual compression in strain elastography, and the other in which an acoustic wave produced by a transducer is used for compression in shear wave elastography (SWE). The standardization of tissue compression can be more precise using the shear-wave technique rather than strain elastography because the compression of the textures can be achieved using repeatable and equalized electronic acoustic waves. Acoustic radiation force impulse (ARFI) elastography is an up-to-date shear-wave technique that can measure the velocity of tissue strain wave scatter perpendicular to compression direction, which is generated by the compression of ARFI. Additional velocity-scale based colorization of ROIs fused in B-mode-images can also be composed $[1,2]$. The main principle of the technique is that the level of tissue stiffness is proportional to the relevant SWV value. The unit for this velocity is 
$\mathrm{m} / \mathrm{s}$, and the velocity equals the square-root of the tissue elasticity [3].

Few studies have been published aiming at investigating the feasibility of SWE for evaluating testicular pathologies, and those studies were limited to neoplastic processes [4-7], infarction $[4,8,9]$, torsion [10], or orchitis [7], and differentiating between these pathologies $[7,11,12]$. Testicular elastography investigations in clinical practice were mostly focused on masses. Increases in stiffness due to neoplastic testicular lesions [4-6] and decreases in stiffness from benign testicular processes, such as orchitis and infarction, have been reported [4].

To the best of our knowledge, there are only a few studies investigating the relationship between testicular strain elastographic evaluation and male fertility $[13,14]$. However, a study demonstrating a correlation between mean testicular stiffness and sperm quantification analyses has not yet been published. In this study, we aimed to evaluate the reliability of mean testicular stiffness quantification using SWE for predicting male fertility potential and for the pre-diagnosis of disorders based on sperm quantification.

\section{Material and methods}

This study was approved by the Van Yuzuncu Yil University Ethics Committee. Written, informed-consent was obtained from all individuals included in the study.

Between February 2014 and April 2015, 137 patients referred to the urology department with a pre-diagnosis of infertility and 10 individuals without any infertility complaint and with normospermia at sperm analysis were initially included in the study. The World Health Organization (WHO) defines infertility as "a disease of the reproductive system defined by the failure to achieve a clinical pregnancy after 12 months or more of regular unprotected sexual intercourse" [15], and the included individuals met these criteria. Thirty four cases with inguinal hernia, hydrocele, acute-past genitourinary trauma, acutechronic scrotal infection, unilateral/bilateral undescended testis, and ejaculation failure (aspermia) due to a potential obstructive issue were excluded. At ultrasonographic (US) evaluation, five patients with a testicular mass and eight patients with atrophic testis (six unilateral and two bilateral) were additionally excluded from the cohort and finally 100 males between the ages of 19-49 with a mean age of $28.77 \pm 6.11$ years were ultimately included.

In all cases grey-scale conventional US, SWE examination, and Doppler US were performed.

\section{$B$-mode US examination}

Scrotal grey-scale US was performed by a radiologist (A.Ya.), with 12 years of experience in conventional US and 4 years of experience in SWE, using 4-9 MHz linear probe (Model 9L4). The machine (Acuson-S2000, Siemens Medical Solutions, Mountain View, CA, USA) parameters were kept constant by using the "factory preset" for "testis/thyroid" evaluation, and the focus level was standardized at the middle portion of the testes.

The volume (measured automatically by using the distances of three largest dimensions of testis) of each testis were recorded. The mean testicular volume of each individual was calculated. The mean testicular volumes of four sub-groups, which were composed upon the semen analyses results, were calculated (see the "Semen Analysis" section below).

\section{SWE examination}

Following B mode US examination, the shear-wave velocity quantification (SWVQ) was performed. An ARFI technique combined with "virtual-touch-tissue-imaging-quantification" (VTIQ) software (Siemens Healthcare, Erlangen, Germany) was used. The transducer was touched laterally to minimize operator-dependent pressure on the testicular tissue. SWVQ was performed individually for each pole in the axial plane, including the $1 / 3$ upper, $1 / 3$ lower, and $1 / 3$ central regions of the testes. The SWVQ method is explained in detail in figure 1. The ROI to form the color-coded SWV map was initially set to completely involve the axial section of the examined testis tissue (upper $1 / 3$ of the right testis in this example); the color-coded SWV maps displaying a spectrum of colors for the related SWV values were generated, and on the left side of this relative SWV-based color-coded map there is a color scale from blue to red representing the SWVs of 0.5 to $6.5 \mathrm{~m} / \mathrm{s}$ (operator-dependent). Then six pin-point-ROIs in VTIQ with dimensions of $1 \mathrm{~mm} \times$ $1 \mathrm{~mm}$ [16] that provided the related SWV values were placed in the shape of a $3 \times 2$ chessboard formation, and the SWV values within the ROIs were automatically quantified in meters per second $(\mathrm{m} / \mathrm{s})$. If there was unclear demarcation of the testicular borders following color-coded shear-wave-velocity map generation, a greyscale shear-wave velocity map accompanied with a synchronized/side-by-side B-mode US image was produced to generate a more accurate positioning of ROIs within the testicular tissue. This technique involved alternative software for SWVQ, which provided an approach to see the pin-point-ROI locations in both grey-scale elastographic and B-mode images simultaneously. While using this software, only a grey-scale elastographic map with similar ranges of 0.5 to $6.5 \mathrm{~m} / \mathrm{s}$ (operator-regulated) could be generated instead of the color-coded map. To reveal a more accurate mean shear-wave velocity value (SWVV) of each testis, quantifications were repeated three times for each region, and 18 SWVVs from each 
$1 / 3$ section of the testis $(6 \times 3=18)$ were obtained (fig 2$)$. The mean SWVVs of upper-central and lower sections of testes were consecutively averaged to calculate the final mean SWVV of each testes. The mean SWVV of the right and left testes was then averaged to determine the mean testicular SWVV for each individual (the mean testicular-SWVV of the sole testis was accepted as the mean SWVV value of the patient in four cases with unilateral orchiectomy).

\section{Doppler US examination}

Doppler US was performed after the SWVQ session to remove any potential effect of straining/Valsalva maneuver on testicular stiffness. The widest enlarged vein and any reflux from the plexus pampiniformis during normal respiration and/or Valsalva maneuver as well as increases in vein diameters during the Valsalva maneuver were determined, if present. Finally, varicocele was classified using the grading system developed by Sarteschi $[17,18]$.

\section{Semen analysis}

Patients were instructed to abstain from sexual activity for 72 hours and to self-collect a semen sample. The semen analysis was obtained twice from each patient and analyzed within 1 hour. The entire sample analysis was conducted by the same lab technician to avoid inter-laboratory variation. Semen volume, sperm count per milliliter (million/ml), total and progressive motility percent (\%), morphology, and vitality percent (\%) were measured. WHO 2010 reference limits $[19,20]$ were used to interpret the results. The patients were divided into four semen analysis based sub-groups (SABSs) according to their semen-analysis parameters: 1) azoospermia (defined as the complete lack of spermatozoa in the ejaculate); 2) oligozoospermia ( $<15$ million sperm/ml); 3) normospermia with decreased motility and agglutination ( $\geq 15$ million sperm $/ \mathrm{ml}$ with progressive motility $<32 \%$ or with progressive + non-progressive motility $<40 \%$ ); and 4) complete normospermia.

\section{Comparison/correlation and Receiver-Operating- \\ Characteristic (ROC) analysis}

Statistical comparisons of mean testicular SWVVs and mean testes volumes between the four SABSs were made. The determination of correlation coefficient values (r) from correlation analysis among the mean testicular SWVVs, testes volumes, patient ages, and sperm-counts were separately evaluated. Additionally, ROC analysis was separately applied to reveal a cut-off value for mean testicular SWVV for differentiating individuals with normal sperm counts from individuals with reduced sperm counts (azo-, olidospermia).

Finally, the comparisons between subgroups with variable grades of varicocele (grades 0 to 3 ) were evaluated in terms of mean testicular SWVVs.

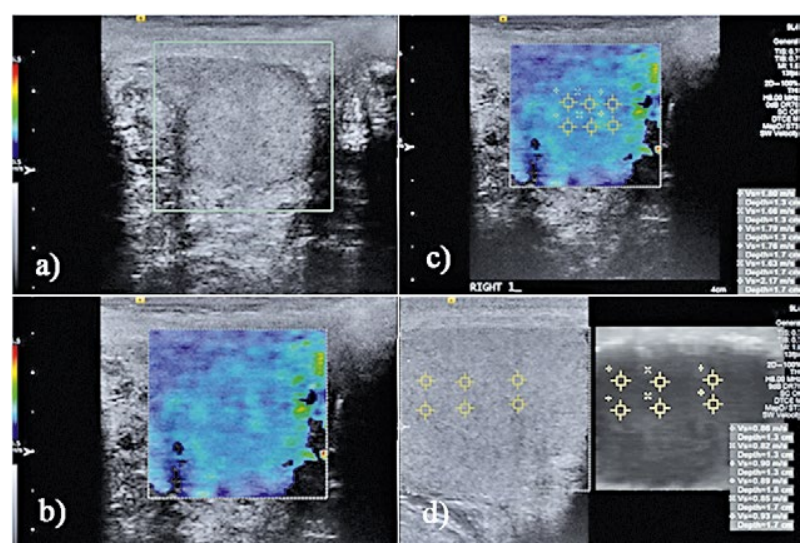

Fig 1. Shear wave velocity (SWV) quantification of the testis with virtual touch tissue imaging quantification (VTIQ) in a 40 year-old male: a) the ROI to form the color-coded SWV map was initially set; b) the color-coded SWV maps displaying a spectrum of colors for the related SWV values were generated; c) six pin-point-ROIs in VTIQ with dimensions of $1 \mathrm{~mm} \times 1$ $\mathrm{mm}$ were placed; d) if there was unclear demarcation of the testicular borders, a grayscale shear-wave-velocity map accompanied with a synchronized/side-by-side B-mode-US image.

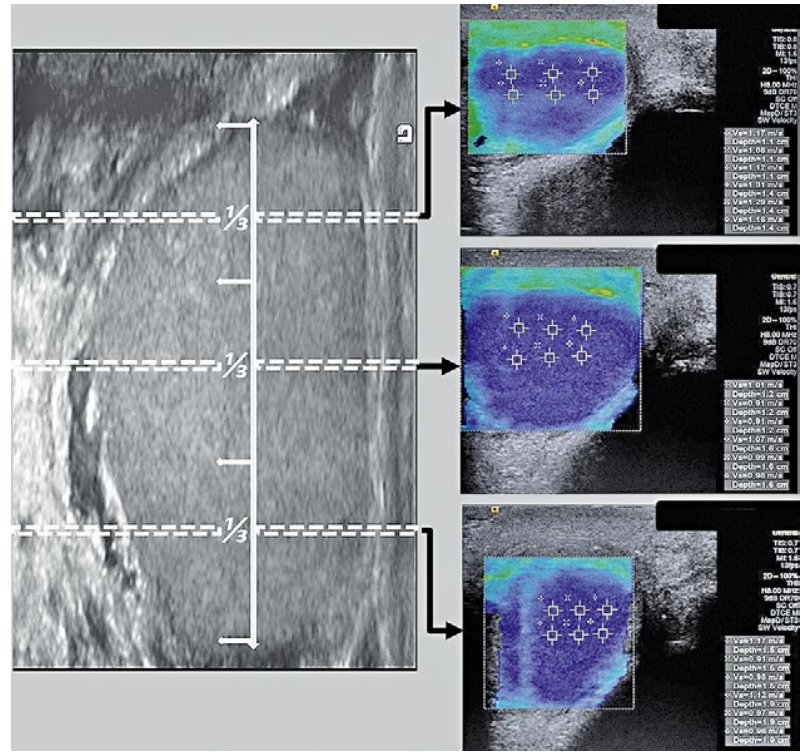

Fig 2. Schematic demonstration of SWV sampling from a testis. To minimize the unavoidable deviations for revealing a more accurate mean SWV value for each testis, quantifications were repeated three times for each $1 / 3$ (upper, central and lower) region, and eighteen SWV values from each $1 / 3$ section of the testis $(6 \times 3=18)$ were obtained.

\section{Intra- and Inter-operator variability analysis in terms of mean testicular SWVQ:}

To determine the intra- and inter-operator variability for mean testicular SWVQ, the mean testicular SWVVs were re-quantified in twenty arbitrary males selected by the initial radiologist (A.Ya.) and by a different ra- 
diologist (A.Yo.) who had six years of expertise in conventional US and two years of experience with SWE. The mean difference and the correlation coefficients (r) between intra- (between A.Ya.'s initial and repeated SVWQ) and inter-operator (between A.Ya.'s initial and A.Yo.'s SVWQ) SWVQ data were determined.

\section{Statistical Analysis}

Descriptive statistics for the studied variables (or characteristics) were presented as the mean, standard deviation (SD), minimum, and maximum values. Oneway analysis of variance (ANOVA) was performed to compare the mean values of these parameters within the described sub-group. Following ANOVA, a Duncan multiple comparison test was used to determine differences among the means. Pearson-correlation-analysis was carried out to examine linear relationships among the variables. The "correlation coefficient" value that indicates the direction and strength of the relationship between two variables was established. A positive value reflected a direct proportional relationship, and a negative value reflected an inverse proportional relationship, and "0" (zero) reflected unrelated variables. To distinguish subgroups according to semen analysis, cut-off values of mean testicular SWV values were determined by ROC analysis. Statistical significance was considered to be $\mathrm{p}<0.05$. SPSS (ver.13) statistical software was used for all statistical computations.

\section{Results}

The distribution of the enrolled patients in the 4 groups, the mean age, mean testicular volume, and mean SWV are detailed in table I. No statistically significant difference between the mean ages of groups was found $(p>0.164)$. The comparison of the mean testis volumes among SABSs revealed that the mean testicular volume in group 1 (azoospermia) was significantly lower than that of the other three groups $(\mathrm{p}<0.001)$. The highest mean SWVV was found in group 1 and the lowest in group 4 $(p<0.05)$. The cut-off values for mean testicular SWVQ to distinguish the population with a normal sperm count (group 3 and group 4) from both azoospermia (group 1) and oligozoospermia (group 2) were determined to be $1.465 \mathrm{~m} / \mathrm{s}(75.0 \%$ sensitivity and $75.0 \%$ specificity) and $1.328 \mathrm{~m} / \mathrm{s}(64.3 \%$ sensitivity and $68.2 \%$ specificity), respectively. Additionally, the cut-off value for the mean testicular SWVQ to differentiate azoospermia (group 1) from oligozoospermia (group 2) was $1.528 \mathrm{~m} / \mathrm{s}$ with $66.7 \%$ sensitivity and $60.7 \%$ specificity.

Overall, 196 testicles from 100 patients (4 patients had unilateral orchiectomy) were examined. For the whole cohort, the mean testicular SWVVs had strong negative correlations with the mean testicular volume $(\mathrm{r}=-0.565, \mathrm{p}<0.01)$ and sperm-count $(\mathrm{r}=-0.399, \mathrm{p}<0.01)$. The mean testicular volume had a strong positive correlation with the sperm-count $(\mathrm{r}=0.491, \mathrm{p}<0.01)$ and moderate with the age $(\mathrm{r}=0.295)$.

We found grade 1-3 varicocele in 11, 6, and 24 testes, respectively (41 testis in 36 patients). No statistical relationship between the presence or the grade of the varicocele and the mean SWVVs of testes was found ( $\mathrm{p}=0.319)$.

The mean differences regarding SWVQ between the intra- and inter-operator measurements were $0.185 \pm 0.125$ $\mathrm{m} / \mathrm{s}$ and $0.139 \pm 0.102 \mathrm{~m} / \mathrm{s}$, respectively. The correlationcoefficients for intra- and inter-operator data in terms of SWVQ were 0.913 and $0.938(\mathrm{p}<0.01)$, respectively.

\section{Discussions}

There have been few physiological studies in which correlations between the testicular volume, testicular SWVV, and age were reported. Di' Anastasi et al found a positive correlation between advanced age and testicular SWVVs and their result was attributed to the increase in testicular stiffness with aging. The age-dependence of testicular stiffness and the decrease of stiffness in the upper pole of the testis compared to the center and lower poles were also reported [21,22]. In our study, a similar negative correlation between the mean testicular volume and advanced age, and a mild positive correlation between the mean testicular SWVV and advanced age were revealed. Additionally, there was a strong negative correlation between the testicular volume and mean testicular SWVV. One of the expected results in our study was the mild negative correlation between advanced age

Table I. The distribution of the patients in 4 groups, the testis volumes, and mean Shear Wave Velocity values

\begin{tabular}{llllll}
\hline Groups & Age (years) & Testis volume $\left(\mathbf{c m}^{3}\right)$ & p & SWVV & p \\
\hline $1(\mathrm{n}=26)$ & $30.88 \pm 6.167$ & $8.127 \pm 5.617$ & 0.001 & $2.325 \pm 1.022 \mathrm{~m} / \mathrm{s}$ & 0.05 \\
$2(\mathrm{n}=30)$ & $27.43 \pm 4.803$ & $12.115 \pm 4.505$ & & $1.512 \pm 0.415 \mathrm{~m} / \mathrm{s}$ & $1.377 \pm 0.363 \mathrm{~m} / \mathrm{s}$ \\
$3(\mathrm{n}=16)$ & $29.60 \pm 6.106$ & $14.63 \pm 4.751$ & & $1.237 \pm 0.208 \mathrm{~m} / \mathrm{s}^{*}$ \\
$4(\mathrm{n}=28)$ & $30.56 \pm 7.330$ & $15.395 \pm 2.959$ & & \\
\hline
\end{tabular}

Study groups were classified by semen analysis (details inside the text). The results are expressed as mean \pm standard deviation (SD); $\mathrm{n}$ - number of patients; SWVV - Shear Wave Velocity values 
and the sperm-count $(\mathrm{r}=-0.124)$, similarly to previously published studies $[23,24]$.

A recent study concluded that SWE can be used to assess testicular tissue hardness both qualitatively and quantitatively, supporting the usefulness of the technique for evaluating various aspects of spermatogenesis disorders [11]. However, this study focused on more controlled experiments with laboratory animals rather than humans. Specifically, the researchers focused on effects of torsion on testicle stiffness and spermatogenesis in rabbits. They found increased stiffness values and decreased spermatogenesis after following different artificial torsion protocols [11]. Although the design of this study differed from ours, a strong negative correlation between the sperm count and mean testicular SWVVs was also established in our study, which suggests that the pathological changes in testicular tissue that led to a decrease in sperm quantity were mostly correlated with an increase in testicular stiffness. According to our results, the testicular tissue hardens and testicular volume decreases with increasing age. The development of tubular involution with increasing age is similar to changes after experimental ischemia, which suggests that vascular lesions may play an important role in age-related testicular atrophy [25]. To test this hypothesis, future studies are required to investigate if the color-coded SWVQ maps used in our study can be used as guides for particular testicular intervention procedures, such as extracting testicular sperm with sufficient quality through "testicular target tissue sampling".

MacLeod et al [26] and Handelsman et al [27] demonstrated that there is a positive correlation with the testes volumes and sperm counts. The same conclusion was drawn in our study and additionally, a significant decrease in testes volume was found in patients with azoospermia and oligozoospermia compared with patients with normal sperm.

We excluded from the study group patients with aspermia due to obstructive causes in order to minimize the potential effects of sperm engorgement on testicular SWVQ. Li et al compared the strain ratio of testes from three cohorts, including a control group and two groups of individuals with azoospermia due to obstructive (aspermia) and non-obstructive etiology [28]. The investigators concluded that a significantly higher rate of average or lower strain values occurred in $81.7 \%$ of testes with non-obstructive azoospermia compared to the values for the obstructive azoospermic (aspermia) testes and control testes $(16.3 \%$ and $15.0 \%, \mathrm{p}<0.001)$. The patients with obstructive azoospermia (aspermia) had only a mildly decreased of the strain ratio (closer to normal), which indicates that testicular tissue can protect itsself in patients with aspermia but not in non-obstructive azoospermia patients. These results are a partial match for our results because the highest SWVVs were encountered in patients with azoospermia (group 1) compared to those of the control group (group 4).

Although the mechanism by which varicocele affects fertility is poorly understood, varicocele is one other potential cause for infertility in males that seems to help damage or kill sperm [29]. Unilateral and unpalpable (sub-clinic) varicocele with reduced testicular volume were determined to be predictors for unsuccessful surgical treatment in terms of post-operative paternity rates $[30,31]$. However, the testicular stiffness was not investigated for the issue of fertility. Although Dede et al revealed decreased stiffness of testicles in patients with variable grades of varicocele [32], there was no statistically significant relationship between the presence or grade of the varicocele and mean testicular SWVV in our study, which was similar to the results of Rocher et al [33]. The effects of varicocele on fertility are mostly based on temperature rise [34] and mild testicular flow disorder [35], which can be fatal for the spermatozoa. These effects are temporary because the sperm count and motility were found to increase after surgical repair [35]. This explanation is supported by the lack of significant change in quantitative testicular stiffness values between the patients with various grades of varicocele (grades 0-4), which was demonstrated by our study (all patients in our study had an initial diagnosis of infertility and possibly had short- or midterm varicocele, yet these details were not investigated for participants enrolled in the study of Dede et al). The difference between the quantification techniques could also be explained by the discrepancy between the study of Dede et al and our study: VTIQ-SWV quantification was used in our study and VTQ-SWV quantification was used by Dede et al. Studies that compare the pre-operative testicular SWVVs and the surgical treatment outcomes in terms of paternity and varicocele are still needed.

There were limitations to our study. The testicular SWVQ was performed using a VTIQ-SWV quantification technique based on multiple pin-point-ROIs, which means that this method had a "sampling" design instead of averaging the whole testicular tissue within a manually created ROI. Future technology developments in SWE will likely be capable of automatically calculating the SWV of a user-defined area or the volume of the inspected tissue and will result in more accurate conclusions. In addition, although it was determined that the pathogenesis of decreased sperm count arises from abnormality in the control mechanism of sperm production at the pre-testicular, testicular, or post testicular level [36], our study 
focused on the testicular level, and there was no investigation of the genital tract or potential pretesticular causes that are characterized by inadequate stimulation of otherwise normal testicles (for example, the serum hormone parameters that can potentially affect the semen analysis results were not examined), and the aspermia cases with obstructive etiology (post-testicular azoospermia in which the sperm are produced but not ejaculated) were excluded. Another limitation of the study is the potential effect of the user-based transducer compression on testistissue, which can lead to errors in SWVQ. In our study, the transducer was touched laterally to the testes instead of perpendicularly to minimize the influence of the user. In this study, the mean testicular SWVQ value for four patients with unilateral orchiectomy was accepted to be the same as the mean testicular value of the sole remaining testis. This decision might affect the accuracy of the results of our study. However, there is an interesting purpose for a new prospective study to determine whether the potential effects of a unilateral orchiectomy on fertility, which could be estimated using pre-operative elastographic examination of the contralateral testis.

\section{Conclusion}

Our study revealed that testicular stiffness quantification using SWE can be an effective method for predicting male fertility and pre-diagnosing infertility disorders, especially those based on sperm quantification. However, elastography is not a complete alternative for semen analysis. In conclusion, SWE, as a non-invasive, easily applicable and repeatable imaging method, has the potential to be a reliable sonographic modality for the diagnosis and follow-up screening of infertility pathologies in male.

\section{Conflict of interest: none}

\section{References}

1. Friedrich-Rust M, Wunder K, Kriener S, et al. Liver fibrosis in viral hepatitis: noninvasive assessment with acoustic radiation force impulse imaging versus transient elastography. Radiology 2009;252:595-604.

2. Zhai L, Palmeri ML, Bouchard RR, Nightingale RW, Nightingale KR. An integrated indenter-ARFI imaging system for tissue stiffness quantification. Ultrason Imaging 2008;30:95-111.

3. Ferraioli G, Tinelli C, Zicchetti M, et al. Reproducibility of real-time shear wave elastography in the evaluation of liver elasticity. Eur J Radiol 2012;81:3102-3106.

4. Aigner F, De Zordo T, Pallwein-Prettner L, et al. Real-time sonoelastography for the evaluation of testicular lesions. Radiology 2012;263:584-589.
5. Pallwein L, Steiner H, Akkad T, Bartsch G, Frauscher F. Real-time elastography for evaluation of testicular masses: initial experience. Eur Urol Suppl 2006;5:96.

6. Pastore AL, Palleschi G, Maceroni P, et al. Correlation between semiquantitative sonoelastography and immunohistochemistry in the evaluation of testicular focal lesions. Cancer Imaging 2014; 14:29.

7. Marsaud A, Durand M, Raffaelli C, et al. Elastography shows promise in testicular cancer detection. Prog Urol 2015;25:75-82.

8. Kantarci F, Cebi Olgun D, Mihmanli I. Shear-wave elastography of segmental infarction of the testis. Korean J Radiol 2012;13:820-822.

9. Patel KV, Huang DY, Sidhu PS. Metachronous bilateral segmental testicular infarction: multi-parametric ultrasound imaging with grey-scale ultrasound, Doppler ultrasound, contrast-enhanced ultrasound (CEUS) and real-time tissue elastography (RTE). J Ultrasound 2014;17:233-238.

10. Sun Z, Xie M, Xiang F, et al. Utility of Real-Time Shear Wave Elastography in the Assessment of Testicular Torsion. PLoS One 2015;10:e0138523.

11. Zhang X, Lv F, Tang J. Shear wave elastography (SWE) is reliable method for testicular spermatogenesis evaluation after torsion. Int J Clin Exp Med 2015;8:7089-7097.

12. Appelbaum L, Gaitini D, Dogra VS. Scrotal ultrasound in adults. Semin Ultrasound CT MR 2013;34:257-273.

13. Schurich M, Aigner F, Frauscher F, Pallwein L. The role of ultrasound in assessment of male fertility. Eur J Obstet Gynecol Reprod Biol 2009;144 Suppl 1:S192-S198.

14. Li M, Du J, Wang ZQ, Li FH. The value of sonoelastography scores and the strain ratio in differential diagnosis of azoospermia. J Urol 2012;188:1861-1866.

15. Zegers-Hochschild F, Adamson GD, de Mouzon J, et al. International Committee for Monitoring Assisted Reproductive Technology (ICMART) and the World Health Organization (WHO) revised glossary of ART terminology, 2009. Fertil Steril 2009;92:1520-1524.

16. Liu H, Zhao LX, Xu G, et al. Diagnostic value of virtual touch tissue imaging quantification for benign and malignant breast lesions with different sizes. Int J Clin Exp Med 2015;8:13118-13126.

17. Liguori G, Trombetta C, Garaffa G, et al. Color Doppler ultrasound investigation of varicocele. World J Urol 2004;22:378-381.

18. Pauroso S, Di Leo N, Fulle I, Di Segni M, Alessi S, Maggini E. Varicocele: ultrasonographic assessment in daily clinical practice. J Ultrasound 2011;14:199-204.

19. Cooper TG, Noonan E, von Eckardstein S, et al. World Health Organization reference values for human semen characteristics. Hum Reprod Update 2010;16:231-245.

20. World Health Organisation. WHO laboratory manual for the examination of human semen and sperm-cervical mucus interaction. Fourth edition. Cambrigde University Press, 1999.

21. Trottmann M, Marcon J, D'Anastasi M, et al. Shear-wave elastography of the testis in the healthy man -determination of standard values. Clin Hemorheol Microcirc 2016;62:273-281. 
22. Marcon J, Trottmann M, D'Anastasi M, et al. 33 Performance of multi-frame shear-wave elastography in the diagnostic work-up of the scrotum. Eur Urol Suppl 2016; $15:$ e33.

23. Cardona Maya W, Berdugo J, Cadavid Jaramillo A. The effects of male age on semen parameters: analysis of 1364 men attending an andrology center. Aging Male 2009; 12:100-103.

24. Hellstrom WJ, Overstreet JW, Sikka SC, et al. Semen and sperm reference ranges for men 45 years of age and older. $\mathrm{J}$ Androl 2006;27:421-428.

25. Paniagua R, Nistal M, Sáez FJ, Fraile B. Ultrastructure of the aging human testis. J Electron Microsc Tech 1991;19:241-260.

26. Macleod J, Wang Y. Male fertility potential in terms of semen quality: a review of the past a study of the present. Fertil Steril 1979;31:103-116.

27. Handelsman DJ, Conway AJ, Boylan LM, Turtle JR. Testicular function in potential sperm donors: normal ranges and the effects of smoking and varicocele. Int $\mathrm{J}$ Androl 1984;7:369-382.

28. Li M, Du J, Wang ZQ, Li FH. The value of sonoelastography scores and the strain ratio in differential diagnosis of azoospermia. J Urol 2012;188:1861-1866.

29. The Practice Committee of the American Society for Reproductive Medicine. Report on varicocele and infertility. Fertil Steril 2008;90:S247-S249.
30. Alshehri FM, Akbar MH, Altwairgi AK, AlThaqufi OJ. Preoperative duplex ultrasound parameters predicting male fertility after successful varicocelectomy. Saudi Med J 2015;36:1439-1445.

31. Donkol RH, Salem T. Paternity after varicocelectomy: preoperative parameters of success. J Ultrasound Med 2007;26: 593-599.

32. Dede O, Teke M, Daggulli M, Utangac M, Bas O, Penbegul N. Elastography to assess the effect of varicoceles on testes: a prospective controlled study. Andrologia 2016;48:257-261.

33. Rocher L, Tanter M, Gennisson J, Criton A, Bellin M, Correas J. Testis Shear Wave Elastography: Preliminary Experience Based on 200 Patients Testis Shear Wave Elastography: Preliminary Experience Based on 200 Patients. Radiological Society of North America 2014 Scientific Assembly and Annual Meeting. Chicago IL. http://archive. rsna.org/2014/14013197.html.

34. Lerchl A, Keck C, Spiteri-Grech J, Nieschlag E, Bergh A. Diurnal variations in scrotal temperature of normal men and patients with varicocele before and after treatment. Int j Androl 1993;16:195-200.

35. Shafik A, Bedeir G. Venous tension patterns in cord veins. I. In normal and varicocele individuals. J Urol 1980;123:383385.

36. Acacio BD, Gottfried T, Israel R, Sokol RZ. Evaluation of a large cohort of men presenting for a screening semen analysis. Fertil Steril 2000;73:595-597. 